\title{
Disorder in quantum critical superconductors
}

\author{
S. Seo ${ }^{1 \dagger}$, Xin Lu' ${ }^{2,3 \dagger}$, J-X. Zhu' ${ }^{2}$, R. R. Urbano ${ }^{2,4}$, N. Curro ${ }^{5}$, E. D. Bauer ${ }^{2}$, V. A. Sidorov ${ }^{2,6}$, L. D. Pham ${ }^{7}$, \\ Tuson Park $^{1 \star}, Z$. Fisk ${ }^{7}$ and J. D. Thompson ${ }^{2 \star}$
}

In four classes of materials-the layered copper oxides, organics, iron pnictides and heavy-fermion compounds-an unconventional superconducting state emerges as a magnetic transition is tuned towards absolute zero temperature, that is, towards a magnetic quantum critical point ${ }^{1}$ (QCP). In most materials, the QCP is accessed by chemical substitution or applied pressure. CeColn $\mathrm{C}_{5}$ is one of the few materials that are 'born' as a quantum critical superconductor ${ }^{2-4}$ and, therefore, offers the opportunity to explore the consequences of chemical disorder. Cadmium-doped crystals of CeColn $\mathrm{Care}_{5}$ a particularly interesting case where $\mathbf{C d}$ substitution induces long-range magnetic order ${ }^{5}$, as in $\mathrm{Zn-doped} \mathrm{copper} \mathrm{oxides}{ }^{6,7}$. Applied pressure globally suppresses the $\mathbf{C d}$-induced magnetic order and restores bulk superconductivity. Here we show, however, that local magnetic correlations, whose spatial extent decreases with applied pressure, persist at the extrapolated QCP. The residual droplets of impurity-induced magnetic moments prevent the reappearance of conventional signatures of quantum criticality, but induce a heterogeneous electronic state. These discoveries show that spin droplets can be a source of electronic heterogeneity and emphasize the need for caution when interpreting the effects of tuning a correlated system by chemical substitution.

Impurities, defects in an otherwise homogeneous host, often are unwanted because their influence can mask intrinsic properties of the host material ${ }^{8}$. Impurities, however, also can be a double-edged sword by providing an avenue to induce interesting new phases of matter and to probe the underlying mechanism of exotic ground states, especially those that emerge from complex interactions in strongly correlated electron materials. In the high-transitiontemperature $\left(T_{\mathrm{c}}\right)$ copper oxide superconductors, the intentional inclusion of small concentrations of non-magnetic impurities, such as $\mathrm{Zn}$, induces magnetism around the impurity site and also enables visualization of the symmetry of the superconducting gap g $^{6,7,9,10}$. Nucleation of a charge density wave in regions surrounding impurity sites is another example of defects revealing the underlying competing phase by disorder ${ }^{11}$. With a growing number of classes of materials that show unusual sensitivity to impurities, understanding and controlling the emergent phases from impurities is an important open issue.

Materials that exhibit an extreme sensitivity to impurities often are near a zero-temperature, second-order phase transition, where quantum critical fluctuations of the associated order parameter diverge in space and time. Theory predicts that when disorder is coupled to these critical fluctuations local regions of an exotic phase can nucleate inside the host and change the nature of the phase transition; indeed, even long-range order of the nucleated phase is possible ${ }^{12-14}$. Nuclear magnetic resonance and scanning tunnelling microscopy have validated these predictions by showing that the host system locally responds to an impurity by creating an extended droplet of the new phase around an impurity ${ }^{6,15}$. Understanding how the local droplet evolves as a function of a control parameter or how the droplet interplays with the host phase, however, is less well known, partly owing to a lack of very pure compounds that are situated sufficiently close to a QCP. The quantum critical superconductor $\mathrm{CeCoIn}_{5}$ provides an ideal opportunity to probe the consequences of impurities on fluctuations of a quantum critical state. It forms readily as exceptionally high-quality single crystals, and the small $(\sim 1 \mathrm{meV})$ characteristic energy of its ground state can be tuned easily with modest applied pressure or magnetic field without introducing additional disorder ${ }^{16-18}$. At ambient pressure, the quantum critical state of CeCoIn ${ }_{5}$, characterized by a linear-in-temperature electrical resistivity above $T_{\mathrm{c}}$ (ref. 3) and logarithmic divergence of the low-temperature electronic specific heat divided by temperature $(C / T)$ (ref. 19), has been interpreted as being due to proximity to a field-induced magnetic QCP (refs 16,20). Replacing one atomic per cent of In by Cd in $\mathrm{CeCo}\left(\operatorname{In}_{1-x} \mathrm{Cd}_{x}\right)_{5}$ reduces $T_{\mathrm{c}}$ from $2.3 \mathrm{~K}(x=0)$ to $T_{\mathrm{c}}=1.2 \mathrm{~K}(x=0.01)$ and induces microscopic coexistence of long-range antiferromagnetic order with $T_{\mathrm{N}}=2.8 \mathrm{~K}$ (refs 5,21). For slightly larger $x$, superconductivity is suppressed completely and only Néel order remains. Introducing these Cd atoms into $\mathrm{CeCoIn}_{5}$ creates defects that produce a response consistent with theoretical expectations ${ }^{12-14}$.

Application of pressure accurately reverses the global effect of Cd substitutions, suppressing the long-range magnetic order and inducing a dome of superconductivity ${ }^{5}$. The fact that the global phase response of $\mathrm{CeCoIn}_{5}$ to cadmium doping can be undone by applied pressure suggests that a quantum critical state should reappear, but the critical point may be hidden by the pressure-induced superconducting state of Cd-doped crystals. This is precisely what is found in the isostructural compound $\mathrm{CeRhIn}_{5}$, which orders antiferromagnetically at atmospheric pressure in the absence of intentionally added impurities. Applying pressure to this correlated electron metal suppresses its long-range antiferromagnetism and induces a dome of bulk superconductivity that hides a QCP that is revealed in an applied magnetic field ${ }^{17,18}$. The pressure evolution of $C / T$ in zero-field is shown in Fig. 1 a as a function of temperature for $\mathrm{CeCoIn}_{5}$ doped with $1 \% \mathrm{Cd}$. Similar results are found for a sample doped with $1.5 \% \mathrm{Cd}$ (see Supplementary Fig. 1). For comparison, we also plot the pressure dependence of $C / T$ of the pristine reference compound $\mathrm{CeRhIn}_{5}$ in Fig. 1b. With applied

\footnotetext{
${ }^{1}$ Department of Physics, Sungkyunkwan University, Suwon 440-746, South Korea, ${ }^{2}$ Los Alamos National Laboratory, Los Alamos, New Mexico 87545, USA, ${ }^{3}$ Center for Correlated Matter and Department of Physics, Zhejiang University, Hangzhou 310027, China, ${ }^{4}$ Instituto de Fisica 'Gleb Wataghin', Universidade Estadual de Campinas-SP, 13083-859, Brazil, ${ }^{5}$ Department of Physics, University of California, Davis, California 95616, USA, ${ }^{6}$ Institute for High Pressure Physics, Russian Academy of Sciences, RU-142190 Troitsk, Moscow, Russia, ${ }^{7}$ Department of Physics, University of California, Irvine, California 92697, USA. †These authors contributed equally to this work. *e-mail: tp8701@skku.edu; jdt@lanl.gov
} 


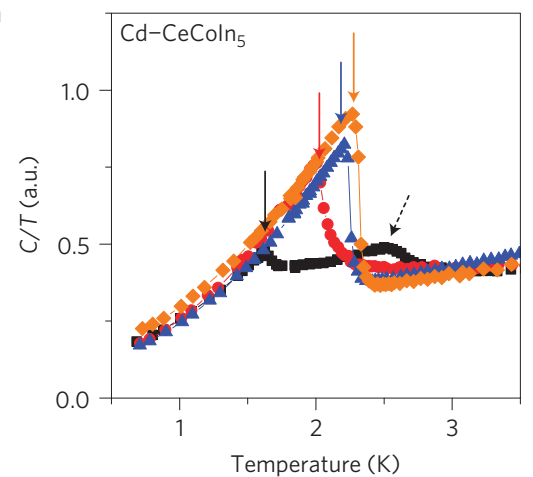

c

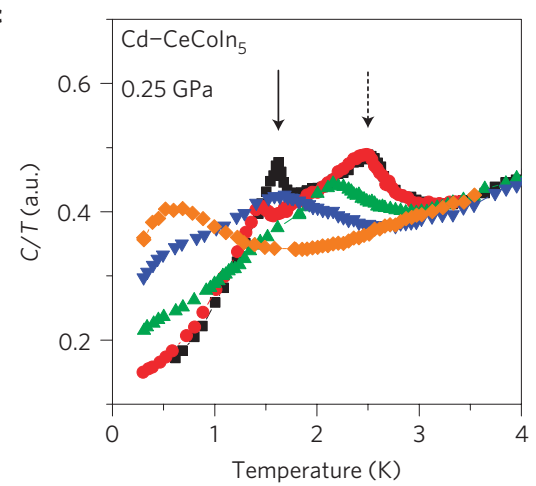

e

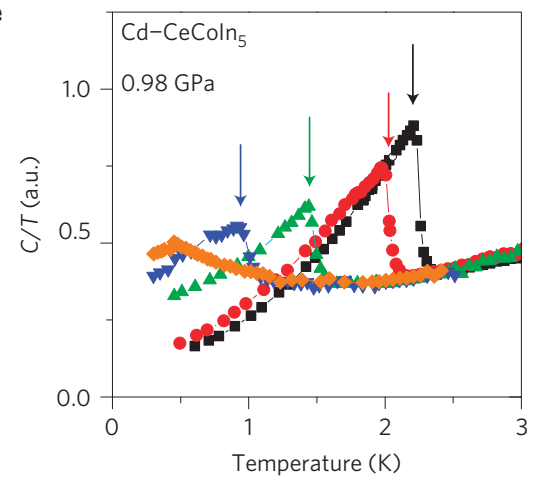

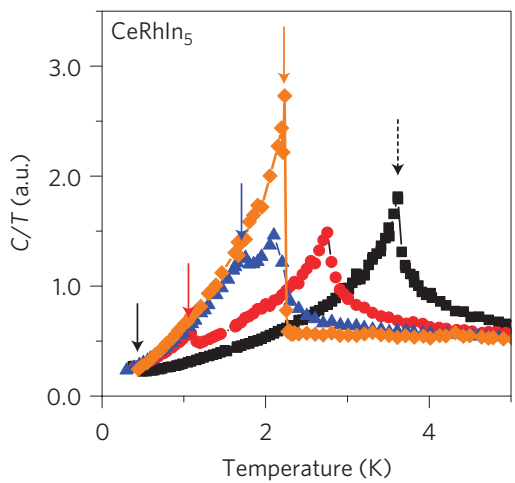

d

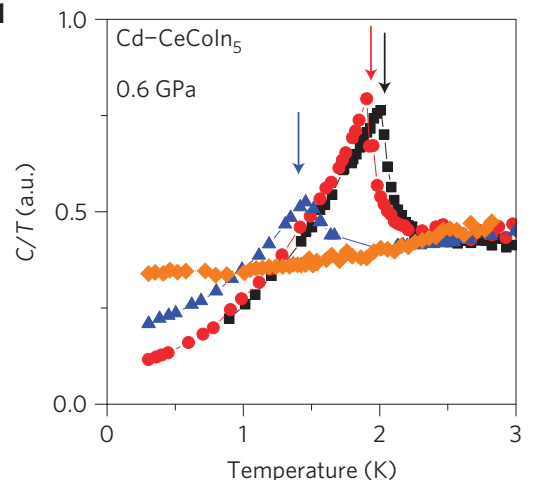

$\mathbf{f}$

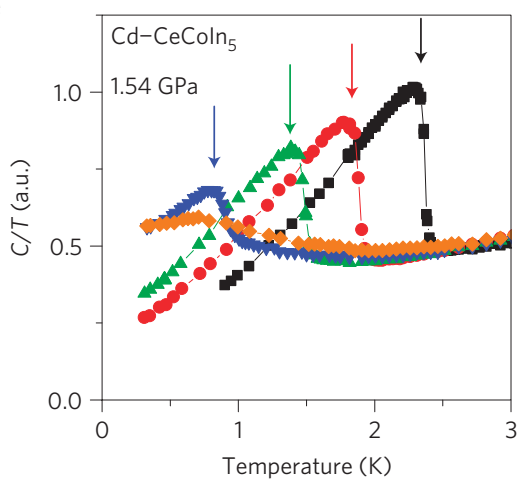

Figure 1 Pressure dependence of the specific heat of $1 \% \mathrm{Cd}$-doped CeColn ${ }_{5}$ and CeRhIn $\mathbf{n}_{5}$ a, Specific heat divided by temperature for $1 \% \mathrm{Cd}$-doped CeColn ${ }_{5}$ in zero applied field and at pressures of 0.25 (black symbols), 0.60 (red), 0.98 (blue) and 1.21 GPa (orange). b, Specific heat divided by temperature for CeRhln 5 at 1.15 (black), 1.51 (red), 1.71 (blue) and $2.05 \mathrm{GPa}$ (orange). In CeRhln ${ }_{5}$, the spin entropy of Ce $4 f$ local moments is transferred completely to the superconducting phase when magnetism is suppressed, resulting in a large $\Delta C / C_{N}>4$ at $T_{C}$ (ref. 18). The qualitative difference between CeRhIn $n_{5}$ and $\mathrm{Cd}$-doped CeColn ${ }_{5}$ exists even though the ordered magnetic moment in both is comparable, $\sim 0.7 \mu_{\mathrm{B}}$. c-f, Dependence on temperature of the specific heat divided by temperature of $1 \% \mathrm{Cd}$-doped CeColn ${ }_{5}$ under magnetic fields and pressures. c, $0.25 \mathrm{GPa}$ at magnetic fields of 0 (black), 1.0 (red), 3.0 (green), 5.0 (blue) and 9.0 T (orange). d, $0.6 \mathrm{GPa}$ at magnetic fields of 0 (black), 1.0 (red), 3.0 (blue) and 5.0 T (orange). e, $0.98 \mathrm{GPa}$ at magnetic fields of 0 (black), 2.0 (red), 4.0 (green), 5.0 (blue) and $5.5 \mathrm{~T}$ (orange). f, $1.54 \mathrm{GPa}$ at magnetic fields of 0 (black), 3.0 (red), 4.0 (green), 5.0 (blue) and 6.0 T (orange). Solid and dashed arrows indicate superconducting and antiferromagnetic transition temperatures, respectively. Values of $C / T$ for different pressures were normalized against each other with an assumption that the entropy recovered at $10 \mathrm{~K}$ is the same for all pressures within each compound. This assumption is proved for $\mathrm{Cd}$-doped $\mathrm{CeColn}_{5}$ as a function of $\mathrm{Cd}$ content at atmospheric pressure ${ }^{5}$.

pressure, $T_{\mathrm{N}}$ is suppressed in both $\mathrm{CeRhIn}_{5}$ and Cd-doped CeCoIn ${ }_{5}$ compounds, and $T_{\mathrm{c}}$ increases. In contrast to CeRhIn $\mathrm{I}_{5}$, however, the discontinuity $\Delta C=C-C_{\mathrm{N}}$, where $C_{\mathrm{N}}$ is the normal-state specific heat at $T_{\mathrm{c}}$, normalized by $C_{\mathrm{N}}, \Delta C / C_{\mathrm{N}} \approx 1.2$ at $T_{\mathrm{c}}$ for Cd-doped $\mathrm{CeCoIn}_{5}$ at $P=1.21 \mathrm{GPa}$. This normalized discontinuity is less than $30 \%$ of the corresponding value in $\mathrm{CeRhIn}_{5}$ at $2.05 \mathrm{GPa}$ or in pure $\mathrm{CeCoIn}_{5}$ at ambient pressure where these materials have nearly identical $T_{\mathrm{c}}$ values.

The effect of a magnetic field on the specific heat of the $1 \% \mathrm{Cd}$-doped $\mathrm{CeCoIn}_{5}$ is summarized in Fig. 1c-f for pressures up to $1.54 \mathrm{GPa}$. For magnetic fields above the critical field $B_{\mathrm{c} 2}$, where superconductivity is completely destroyed, $C / T$ at low temperatures decreases with decreasing temperature typical of a non-critical metal. At $0.98 \mathrm{GPa}$ and $6.2 \mathrm{~T}$, which is just above the upper critical field $B_{\mathrm{c} 2}$ at this pressure, there is a slight upturn in $C / T$ at the lowest temperatures. This weak upturn, however, is distinct from undoped $\mathrm{CeCoIn}_{5}$, where $C / T$ logarithmically diverges with decreasing temperature near $B_{\mathrm{c} 2}$ (ref. 16) and from $\mathrm{CeRhIn}_{5}$ at its quantum critical pressure, where $C / T$ also diverges once superconductivity is suppressed by a magnetic field ${ }^{18}$. The prominent lack of a divergence in $C / T$ of the Cd-doped compound indicates that quantum critical behaviour is avoided. 
a

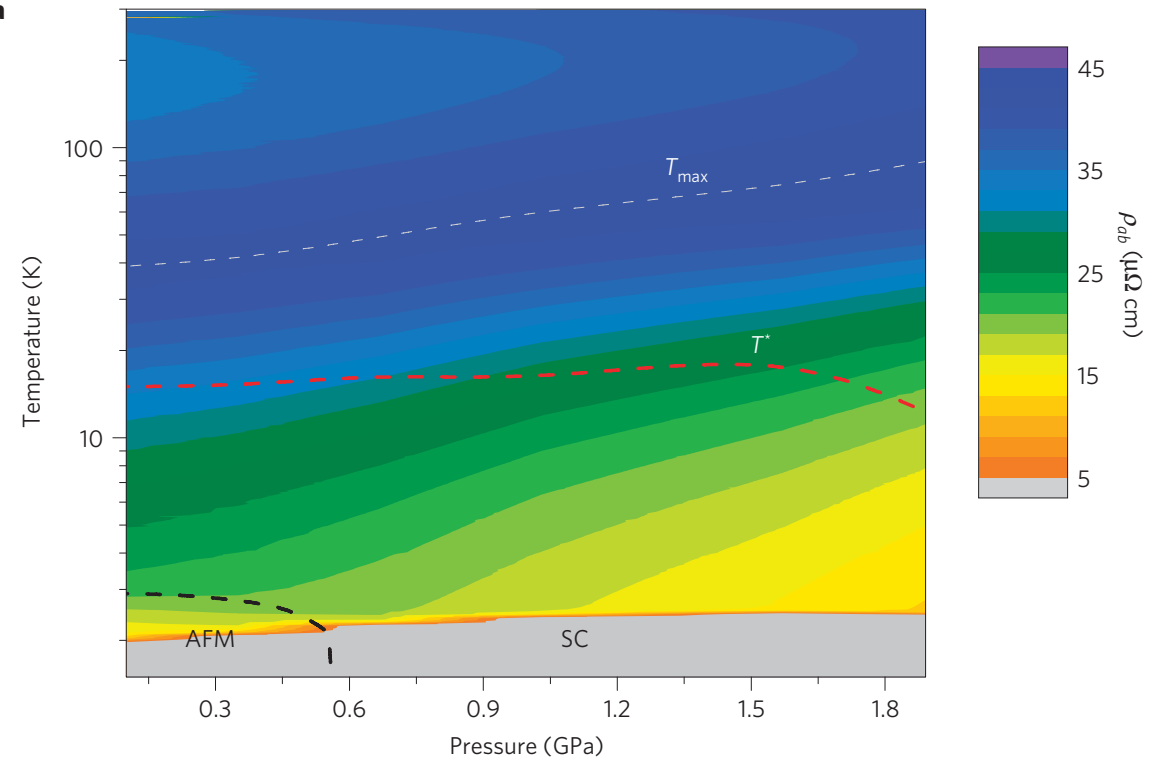

b

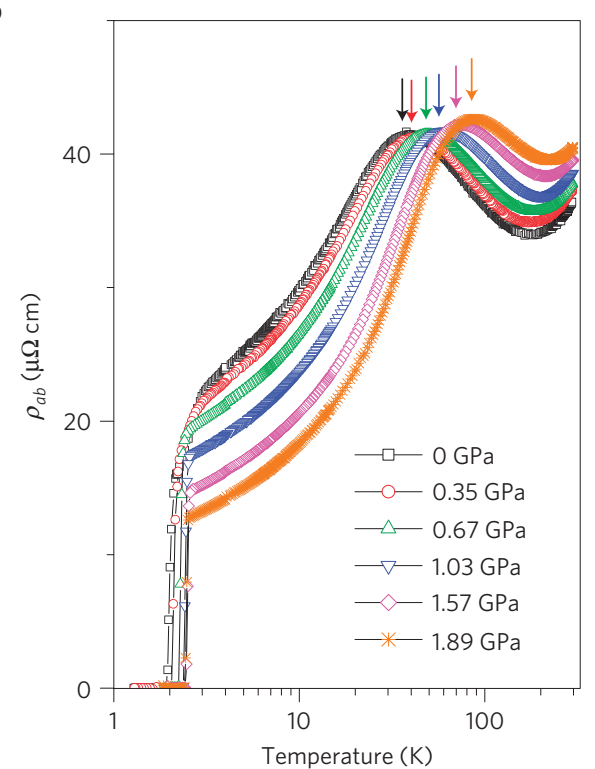

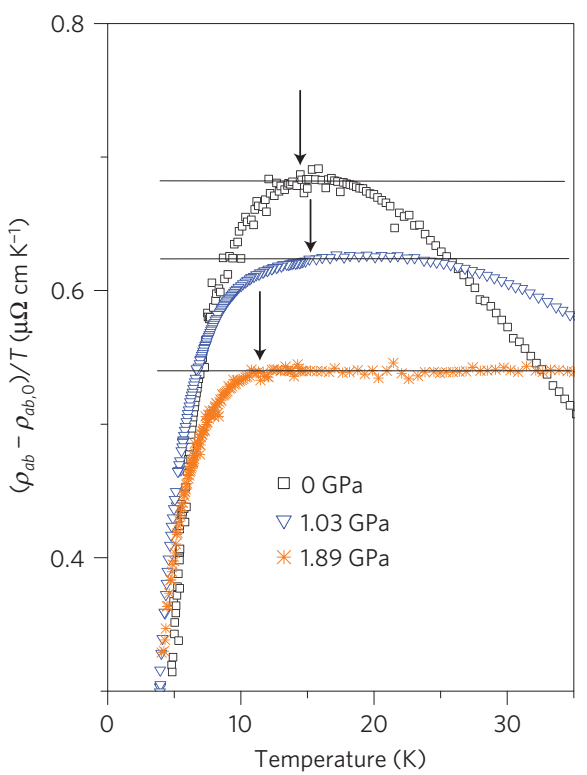

Figure 2 | Electrical resistivity of $1 \% \mathbf{C d}$-doped $\mathbf{C e C o l n} \mathbf{n}_{\mathbf{5}}$ under pressure. a, Contour map of the electrical resistivity $\left(\rho_{a b}\right)$ within the Ce-In plane plotted in the pressure-temperature plane. $T_{\max }$ is a temperature where the resistivity reaches a maximum, and $T^{*}$ is the temperature below which the resistivity deviates from a $T$-linear dependence. An antiferromagnetic state coexists with superconductivity for $P<0.5 \mathrm{GPa}$. $\mathbf{b}$, In-plane resistivity $\rho_{a b}$ as a function of temperature on a semi-logarithmic scale for representative pressures. Arrows mark the evolution of $T_{\max }$ with pressure. The temperature $T_{\max }$ at which $\rho(T)$ is a maximum occurs at $34 \mathrm{~K}$ at ambient pressure and increases linearly with increasing pressure, which is typical of strongly correlated Ce-based materials, such as pure CeColn 5 , and reflects a pressure-induced increase of the hybridization between ligand electrons and the periodic array of $\mathrm{Ce}$ $4 f$-electrons. c, Resistivity, with the residual $T=0$ value subtracted, divided by temperature, plotted against temperature for representative pressures. $T^{*}$ is marked by arrows.

Supporting this lack of quantum criticality from specific heat measurements, the electrical resistivity $\rho$ of $1 \% \mathrm{Cd}$-doped $\mathrm{CeCoIn}_{5}$ also does not show an anomalous temperature dependence characteristic of proximity to a QCP. Isothermal cuts of the low-temperature electrical resistivity as a function of pressure (Fig. 2a) show that $\rho(P)$ decreases monotonically for $T \geq T_{\mathrm{c}}$ across the critical pressure $P_{\mathrm{cl}}(=0.5 \mathrm{GPa})$, where $T_{\mathrm{N}}$ becomes equal to $T_{\mathrm{c}}$, and $P_{\mathrm{c} 2}(=1.0 \mathrm{GPa})$, the extrapolated critical point where $T_{\mathrm{N}}$ extrapolates to $0 \mathrm{~K}$ inside the superconducting dome. Further, Fig. 2c shows a linear- $T$ resistivity over a temperature range between 12 and $35 \mathrm{~K}$ at $1.89 \mathrm{GPa}$, but it becomes sub-linear in $T$ at lower temperatures, in contrast to expectations of quantum criticality and the $T$-linear $\rho(T)$ in pristine $\mathrm{CeCoIn}_{5}$ that extends to $T_{\mathrm{c}}$ (ref. 3). This sub-linear temperature dependence of the resistivity demonstrates that the spectrum of spin excitations has changed with doping such that the scattering rate below $T^{*}$ is reduced relative to a continuation of the $T$-linear resistivity to lower temperatures, a reduction in scattering arising from possible short-range magnetic correlations.

The large jump in specific heat at $T_{c}$ in pure $\mathrm{CeCoIn}_{5}$ and in CeRhIn 5 at its pressure-tuned QCP is a consequence of the huge quantum disordered entropy of magnetic fluctuations that is recovered in the zero-temperature limit when superconductivity is suppressed by a magnetic field. In contrast, the specific 
a

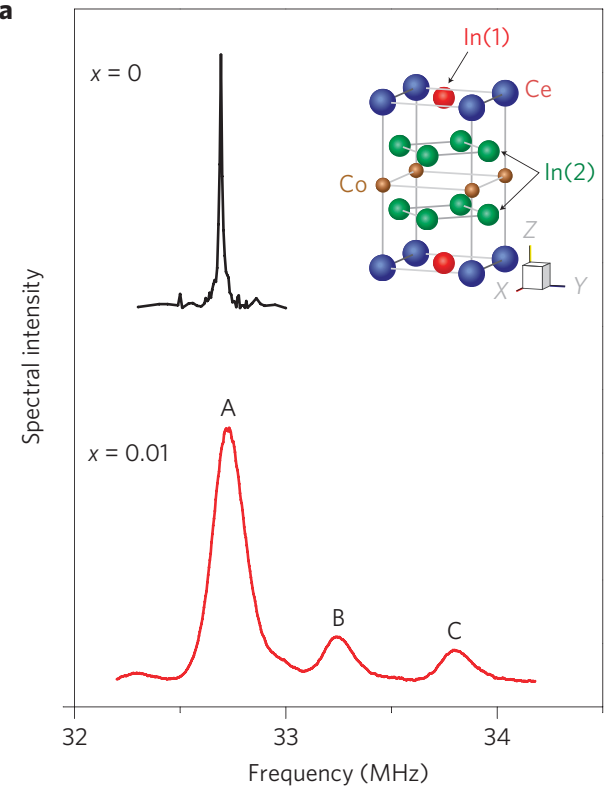

b

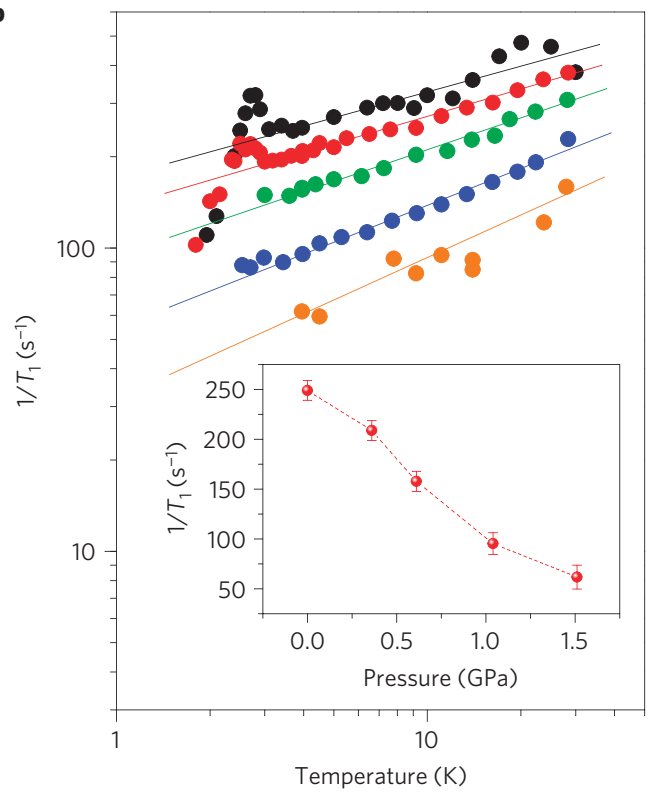

C
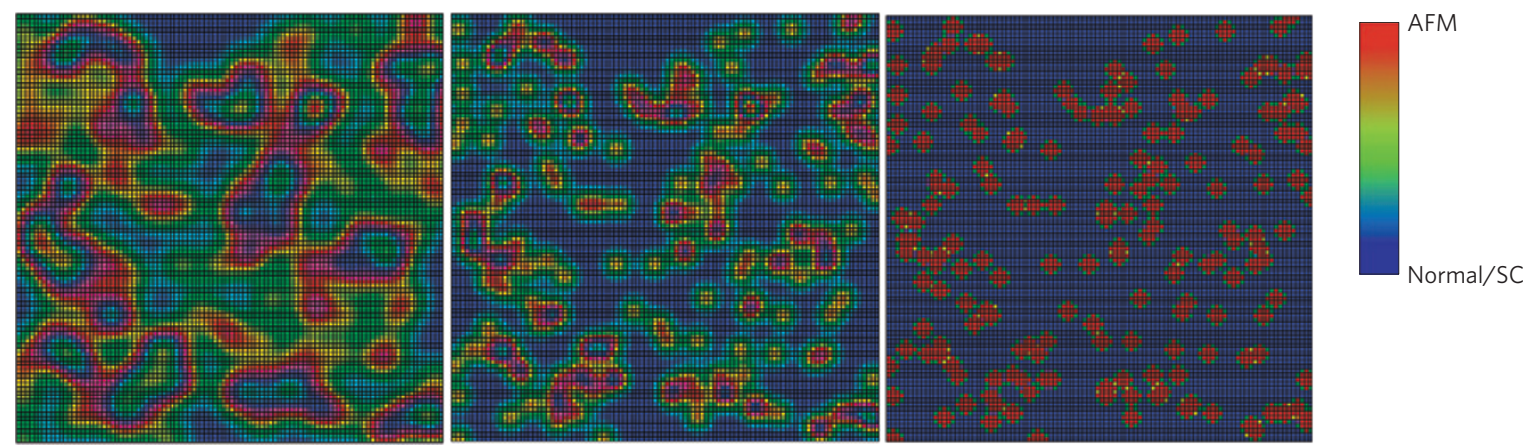

Pressure

Figure 3 | Spin-lattice relaxation rate of $1 \%$ Cd-doped CeColn 5 under pressure. $\mathbf{a}^{115} \operatorname{In} N Q R$ spectra of the $\ln (1)$ site at ambient pressure. ${ }^{115}$ In NQR spectra of $\mathrm{CeColn}_{5}$ and $1 \% \mathrm{Cd}$-doped $\mathrm{CeColn}_{5}$ are shown in the upper and lower parts of the panel. Indium sites in the unit cell of CeColn $n_{5}$ are indicated in the inset. In the $\mathrm{Cd}$-doped crystals, additional peaks $\mathrm{B}$ and $\mathrm{C}$ appear at higher frequencies, and the original peak $\mathrm{A}$ is broadened and moved slightly to higher frequency. $\mathbf{b}$, Dependence on temperature of the spin-lattice relaxation rate $1 / T_{1}$ of $\mathrm{CeCo}\left(\ln _{0.99} \mathrm{Cd}_{0.01}\right)_{5}$. Solid lines are guides to the eyes for pressures of 1 bar (black), 0.36 (red), 0.61 (green), 1.04 (blue) and $1.51 \mathrm{GPa}$ (orange). The overall decrease in $1 / T_{1}$, even at high temperatures, is found in pristine $\mathrm{CeColn}_{5}$ (ref. 26). The inset shows $1 / T_{1}$ at $4 \mathrm{~K}$ as a function of pressure. Error bars describe the uncertainty in $1 / T_{1}$. c, Schematic illustration of the dependence on pressure of the size of magnetic droplets. The $\mathrm{Cd}$ atoms that replace $\mathrm{In}$ in $\mathrm{CeColn} 5$ nucleate antiferromagnetic droplets surrounding the $\mathrm{Cd}$ site. The average distance between the droplets, which is approximately five lattice spacings at $0.75 \% \mathrm{Cd}$ doping, is independent of pressure, but their spatial extent shrinks with pressure. For a pressure above $P_{\mathrm{c} 1}$ (the middle panel), the magnetic correlation length becomes shorter than inter-droplet spacing, leading to suppression of the long-ranged antiferromagnetic order. AFM, antiferromagnetic; SC, superconducting.

heat jump in Cd-doped $\mathrm{CeCoIn}_{5}$ is smaller, even when these crystals are tuned to their putative critical point. This small jump, together with the absence of a signature for a diverging specific heat in a magnetic field and $T$-linear $\rho(T)$ to $T_{c}$, indicates that the spectrum of magnetic fluctuations has changed in response to the presence of $\mathrm{Cd}$ impurities. When very dilute concentrations of non-magnetic impurities are added to $\mathrm{CeCoIn}_{5}$, a resonance in the electronic density of states develops from unitary scattering of the highly correlated band of conduction electrons by the local defect ${ }^{22}$. In this process, the non-magnetic defect acquires the magnetic character of a spin $S=1 / 2$ Kondo impurity. At higher concentrations, local droplets of static magnetic order nucleate with a typical extent of a few lattice parameters ${ }^{21}$, and when these droplets overlap, long-range antiferromagnetic order develops. Although magnetic fluctuations may be suppressed locally through the nucleation of spin droplets around the Cd impurities, they should not be affected significantly at distances removed from these impurities where there is no static magnetism. Apparently, sufficient spectral weight remains in the unaffected fluctuations to produce a superconducting transition only slightly lower than that of pure $\mathrm{CeCoIn}$.

Figure 3a shows ${ }^{115}$ In nuclear quadrupolar resonance (NQR) spectra of the In(1) site for CeCoIn 5 and $1 \%$ Cd-doped CeCoIn 5 . Three peaks A, B and C are observed in Cd-doped crystals ${ }^{21}$, where peak A probes the bulk of the unit cells as in pristine $\mathrm{CeCoIn}_{5}$. The existence of $\mathrm{B}$ and $\mathrm{C}$ peaks underscores the presence of a minority of In sites in an electronic environment not present in undoped $\mathrm{CeCoIn}_{5}$. Cd impurities significantly broaden the $\operatorname{In}(1)$ NQR spectra owing to the distribution of 
local electric field gradients. Figure $3 b$ shows the temperature dependence of the spin-lattice relaxation rate $1 / T_{1}$ measured at peak A of $1 \% \mathrm{Cd}$-doped $\mathrm{CeCoIn}_{5}$ under pressures to $1.51 \mathrm{GPa}$. At ambient pressure, $1 / T_{1}$ is identical to the pure compound and follows a $T^{1 / 4}$ temperature dependence in the disordered state, which is expected for a system in close proximity to an antiferromagnetic QCP (refs 21,23). The observation that $1 / T_{1}$ is a strong function of pressure yet independent of $\mathrm{Cd}$ doping provides microscopic evidence that pressure is not the reverse of doping. Taken with the specific heat data, these data imply that the electronic response to $\mathrm{Cd}$ doping is strongly inhomogeneous. As pressure tunes the system away from longrange magnetic order, the spin-spin correlation length, $\xi$, and therefore the size of the droplets, should decrease. In the temperature range around $T_{\mathrm{N}}(P=0)$, this is reflected in a rapid decrease of the relaxation rate $1 / T_{1} \propto T \xi^{\alpha}$ (refs 23-27) that is plotted in Fig. 3b. These NQR results provide microscopic evidence for the existence of spin droplets even when global long-range antiferromagnetic order is suppressed completely for pressures above $P_{\mathrm{cl}}$.

The nucleation and pressure evolution of spin droplets can be understood within the framework of a theoretical model that considers the competition between antiferromagnetism and $d$-wave superconductivity ${ }^{13}$ (details of the model are discussed in Supplementary Information). In the absence of impurities, this model gives negligible spectral weight in the magnetic channel and homogeneous $d$-wave superconductivity near a QCP. When a unitary scattering impurity is introduced, however, superconductivity is suppressed strongly around the defect but recovers over a distance of a coherence length away from the impurity. Concurrently, magnetic order is depressed at the impurity but is a maximum at the nearest Ce neighbour and decays over many lattice constants. As shown in Supplementary Fig. 4a,b, the superconducting order is robust against a change in effective bandwidth (or the external pressure), but the magnetic order is very sensitive. A slight increase in bandwidth rapidly dampens the longrange oscillatory magnetization and the amplitude of the nearestneighbour magnetic order parameter. For a dilute concentration of impurity centres or at sufficiently wider bandwidths (higher pressures), there will be only magnetic droplets, but for a narrower bandwidth or higher concentration of scattering centres, magnetic correlations between sites induce global magnetic order. Predictions of this model are illustrated schematically in Fig. 3c.

The lack of signatures for quantum critical behaviour in Cddoped $\mathrm{CeCoIn}_{5}$ manifests a new mechanism for the coexistence of magnetism and superconductivity and provides a different perspective for interpreting the response to disorder in other strongly correlated superconductors near a zero-temperature magnetic instability. As this work shows, tuning a system with or by disorder to a presumed magnetic QCP does not necessitate a quantum critical response and associated spectrum of quantum fluctuations. Although not all impurities may be unitary scatterers, the likelihood is high that they will be strong scatterers if the bandwidth of the host material is sufficiently narrow, as it is in many strongly correlated heavy-fermion compounds, such as $\mathrm{CeCoIn}_{5}$. The freezing of magnetic quantum fluctuations around impurity sites has broader consequences for the nature of electronic heterogeneity that is common to classes of strongly correlated electron systems.

\section{Methods}

Single crystals of $\mathrm{CeCo}\left(\operatorname{In}_{1-x} \mathrm{Cd}_{x}\right)_{5}$ were synthesized by a standard In-flux technique and their basic physical properties were reported previously ${ }^{5}$. Electrical resistivity, specific heat and NQR measurements were performed under pressure for samples with Cd concentrations $x=0.01$ and 0.015 , where the concentration $x$ is determined by microprobe measurements. A quasi-hydrostatic pressure environment was achieved in a $\mathrm{Be}-\mathrm{Cu} / \mathrm{NiCrAl}$ hybrid clamp-type cell with silicone oil as the transmitting medium and pressure in the cell was determined at low temperatures by inductive measurements of a change in the superconducting transition temperature of $\mathrm{Sn}$ that was placed inside the cell ${ }^{28}$. Specific heat measurements were performed by an a.c. calorimetric technique, where the voltage in $\mathrm{Au} / \mathrm{Fe}(0.07 \%)$ thermocouple wire, attached to one facet of the crystal, was monitored and converted to a temperature change that is inversely proportional to the specific heat. Details of the a.c. calorimetric technique can be found elsewhere ${ }^{29}$. A standard four-probe technique was employed to measure the electrical resistivity using an LR700 resistance bridge. The spin-lattice relaxation time $1 / T_{1}$ at the A site, which is associated with the bulk high-symmetry $\operatorname{In}(1)$ sites, was obtained at zero field by measuring the NQR of a single-crystalline sample.

Received 20 December 2012; accepted 23 October 2013; published online 22 December 2013

\section{References}

1. Taillefer, L. Scattering and pairing in cuprate superconductors. Annu. Rev. Condens. Matter Phys. 1, 51-70 (2010).

2. Stockert, O. et al. Magnetically driven superconductivity in $\mathrm{CeCu}_{2} \mathrm{Si}_{2}$. Nature Phys. 7, 119-124 (2011).

3. Sidorov, V. A. et al. Superconductivity and quantum criticality in $\mathrm{CeCoIn}_{5}$. Phys. Rev. Lett. 89, 157004 (2002).

4. Matsumoto, Y. et al. Quantum criticality without tuning in the mixed valence compound $\beta-\mathrm{YbAlB}_{4}$. Science 331, 316-319 (2011).

5. Pham, L. D., Park, T., Maquilon, S., Thompson, J. D. \& Fisk, Z. Reversible tuning of the heavy-fermion ground state in CeCoIn ${ }_{5}$. Phys. Rev. Lett. 97, 056404 (2006).

6. Alloul, H., Mendels, P., Casalta, H., Marucco, J. F. \& Arabski, J. Correlation between magnetic and superconducting properties of $\mathrm{Zn}$-substituted $\mathrm{YBa}_{2} \mathrm{Cu}_{3} \mathrm{O}_{6+x}$. Phys. Rev. Lett. 67, 3140-3143 (1991).

7. Kimura, H., Kofu, M., Matsumoto, Y. \& Hirota, K. Novel in-gap spin state in Zn-doped $\mathrm{La}_{1.85} \mathrm{Sr}_{0.15} \mathrm{CuO}_{4}$. Phys. Rev. Lett. 91, 067002 (2003).

8. Takagi, S. et al. No evidence for small-moment antiferromagnetism under ambient pressure in $\mathrm{URu}_{2} \mathrm{Si}_{2}$ : Single-crystal ${ }^{29} \mathrm{Si}$ NMR study. J. Phys. Soc. Jpn 76, 033708 (2007).

9. Balatsky, A. V., Vekhter, I. \& Zhu, J-X. Impurity-induced states in conventional and unconventional superconductors. Rev. Mod. Phys. 78, 373-433 (2006).

10. Alloul, H., Bobroff, J., Gabay, M. \& Hirschfeld, P. J. Defects in correlated metals and superconductors. Rev. Mod. Phys. 81, 45-108 (2009).

11. Weitering, H. H. et al. Defect-mediated condensation of a charge density wave. Science 285, 2107-2110 (1999)

12. Millis, A. J., Morr, D. K. \& Schmalian, J. Local defect in metallic quantum critical systems. Phys. Rev. Lett. 87, 167202 (2001).

13. Zhu, J-X., Martin, I. \& Bishop, A. R. Spin and charge order around vortices and impurities in high- $T_{c}$ superconductors. Phys. Rev. Lett. 89, 067003 (2002).

14. Andersen, B. M., Hirschfeld, P. J., Kampf, A. P. \& Schmid, M. Disorder-induced static antiferromagnetism in cuprate superconductors. Phys. Rev. Lett. 99, 147002 (2007)

15. Pan, S. H. et al. Imaging the effects of individual zinc impurity atoms on superconductivity in $\mathrm{Bi}_{2} \mathrm{Sr}_{2} \mathrm{CaCu}_{2} \mathrm{O}_{8+\mathrm{d}}$. Nature 403, 746-750 (2000).

16. Bianchi, A., Movshovich, R., Vekhter, I., Pagliuso, P. G. \& Sarrao, J. L. Avoided antiferromagnetic order and quantum critical point in $\mathrm{CeCoIn}_{5}$ Phys. Rev. Lett. 91, 257001 (2003).

17. Knebel, G., Aoki, D., Braithwaite, D., Salce, B. \& Flouquet, J. Coexistence of antiferromagnetism and superconductivity in $\mathrm{CeRhIn}_{5}$ under high pressure and magnetic field. Phys. Rev. B 74, 020501 (2006).

18. Park, T. et al. Hidden magnetism and quantum criticality in the heavy fermion

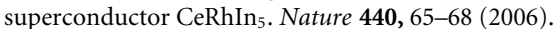

19. Petrovic, C. et al. Heavy-fermion superconductivity in $\mathrm{CeCoIn}_{5}$ at $2.3 \mathrm{~K}$ J. Phys. Condens. Matter 13, L337-L342 (2001).

20. Paglione, J. et al. Nonvanishing energy scales at the quantum critical point of CeCoIn 5 . Phys. Rev. Lett. 97, 106606 (2006).

21. Urbano, R. R. et al. Interacting antiferromagnetic droplets in quantum critical CeCoIn. . Phys. Rev. Lett. 99, 146402 (2007).

22. Bauer, E. D. et al. Electronic inhomogeneity in a Kondo lattice. Proc. Natl Acad. Sci. USA 108, 6857-6861 (2011).

23. Ishigaki, A. \& Moriya, T. Nuclear magnetic relaxation around the magnetic instabilities in metals. J. Phys. Soc. Jpn 65, 3402-3403 (1996).

24. Kohori, Y. et al. ${ }^{115}$ In NQR studies of CeRhIn ${ }_{5}$ and $\mathrm{CeCoIn}_{5}$ under high pressure. J. Alloys. Compd. 408, 51-53 (2006).

25. Moriya, T. \& Ueda, K. Spin fluctuations and high temperature superconductivity. Adv. Phys. 49, 555-606 (2000).

26. Millis, A. J., Monien, H. \& Pines, D. Phenomenological model of nuclear relaxation in the normal state of $\mathrm{YBa}_{2} \mathrm{Cu}_{3} \mathrm{O}_{7}$. Phys. Rev. B 42, 167-178 (1990).

27. Wolfe, P. \& Abrahams, E. Quasiparticles beyond the Fermi liquid and heavy fermion criticality. Phys. Rev. B 84, 041101(R) (2011). 
28. Eiling, A. \& Schilling, J. S. Pressure and temperature dependence of electrical resistivity of $\mathrm{Pb}$ and $\mathrm{Sn}$ from $1-300 \mathrm{~K}$ and $0-10 \mathrm{GPa}$-use as continuous resistive pressure monitor accurate over wide temperature range; superconductivity under pressure in Pb, Sn, and In. J. Phys. F 11, 623-639 (1981).

29. Kraftmakher, Y. Modulation calorimetry and related techniques. Phys. Rep. 356, 1-117 (2002).

\section{Acknowledgements}

We thank F. Ronning, M. Vojta and J. Shim for helpful discussions. Work at Los Alamo was performed under the auspices of the US Department of Energy, Office of Science,

Division of Materials Science and Engineering and supported in part by the Los Alamos LDRD program. Work at SKKU is supported by an NRF grant funded by the Korean Ministry of Education, Science \& Technology (MEST) (No. 2012R1A3A2048816 \& 220 2011-1-C00014). R.R.U. acknowledges FAPESP (No. 2012/05903-6). V.A.S. acknowledges support by RFBR Grant 12-02-00376. X.L. acknowledges NSFC (No. 11374257).

\section{Author contributions}

All authors discussed the results and commented on the manuscript. S.S. and X.L. performed the measurements and contributed equally to this work. R.R.U., V.A.S. and N.C. performed and analysed NQR experiments. E.D.B., L.D.P. and Z.F. provided samples, J-X.Z. performed theoretical calculations and T.P. and J.D.T. wrote the manuscript with input from all authors.

\section{Additional information}

Supplementary information is available in the online version of the paper. Reprints and permissions information is available online at www.nature.com/reprints. Correspondence and requests for materials should be addressed to T.P. or J.D.T.

\section{Competing financial interests}

The authors declare no competing financial interests. 